\title{
Influence of manipulated category knowledge on prototype classification and recognition
}

\author{
DONALD HOMA, BARBARA GOLDHARDT, LORI BURRUEL-HOMA, and J. CARSON SMITH \\ Arizona State University, Tempe, Arizona
}

\begin{abstract}
The recognition and classification of category members was explored, following a variable number of learning trials. In Experiment 1, subjects received 1 or 9 learning trials, followed by a recognition-then-classification test containing old, new, prototype, and foil patterns. In Experiment 2 , subjects received 1,6 , or 12 trials, and made either classification or recognition judgments. In each experiment, classification accuracy for all item types was at near-chance performance after a single trial but steadily increased with increased learning trials. On the transfer test, oldness judgments were highest for the category prototype after a single trial. However, with increased learning trials, oldness judgments increased for old instances and decreased for the category prototype and new instances. We suggest that false recognition of the category prototype, especially after a single learning trial, need not reflect an abstraction process. We discuss the possibility that an abstracted prototype may emerge with additional learning as an unfamiliar, ideal point.
\end{abstract}

This research focuses on false recognitions of category prototypes as old following differing numbers of learning trials with multiple categories. The tendency for prototypes to be rated as old when they were not presented during training is one of the puzzles of category learning.

We know that categorical knowledge, at least for illdefined categories (Homa, 1984; Neisser, 1967), is dynamic in the sense that its representation is modifiable by the experiences that define it. For example, accuracy of classification of the category prototype, as well as other novel instances of that category, is enhanced when the category is defined in the learning phase by many, rather than few, instances (e.g., Homa, Cross, Cornell, Goldman, \& Shwartz, 1973), when learning trials are increased (Homa, Dunbar, \& Nohre, 1991), and when training pattern distortion is mixed or variable, rather than uniform and minimal (Homa \& Vosburgh, 1976; Posner \& Keele, 1968). Consistent with this view is the finding that the empirical prototype (the pattern that is the average of the training set of instances) is classified more accurately than any other transfer pattern, including the objective prototype, especially when the category size is increased (Breen \& Schvaneveldt, 1986).

Numerous models of classification capture the evolving property of categorical knowledge by positing integrative mechanisms that operate on the stimulus inputs that define the category (e.g., Knapp \& Anderson, 1984; McClelland \& Rumelhart, 1986). In these models, the cat-

Portions of Experiments 1 and 2 were presented to the Western Psychological Association, in Reno, Nevada, April 1989. We would like to thank Tony Mandts for reading an earlier version of this manuscript. Requests for reprints should be addressed to D. Homa, Department of Psychology, Arizona State University, Tempe, AZ 85287. egory prototype is simply the pattern that is central to the activations of individual traces.

These models appear to be well suited to account for another finding in categorization that is the focus of the present study: The category prototype often receives a higher oldness rating on a subsequent recognition test than do other transfer patterns, including the training instances, even though the prototype is novel (e.g., Franks \& Bransford, 1971; Metcalfe \& Fisher, 1986; Neumann, 1977; Solso \& McCarthy, 1981a, 1981b; Solso \& Raynis, 1979). A few exceptions have been noted. For example, when the subject is informed that the learning instances will contain only certain features, subsequent false recognition of the category prototype, which contains a novel collection of averaged feature values, is reduced; omitting these instructions reinstates the effect (Neumann, 1977). ${ }^{1}$ Connectionist models of classification (Knapp \& Anderson, 1984; McClelland \& Rumelhart, 1986) predict the high rate of false recognition of the category prototype if the training patterns are sufficiently similar to the prototype. This is because the individual activations of each training pattern summate strongly at the centroid of the training patterns, which corresponds, in general, to the prototype. Thus, these summed activations of the prototypes exceed those of any individual old pattern, at least if sufficient numbers of patterns are used. This view predicts that the summed activations would yield false recognitions of the prototypes.

Nonetheless, the assertion that prototype abstraction is revealed by false alarming to the category prototype is bothersome for a number of reasons. First, the effect is invariably reported in single-trial paradigms, where category formation should be low. In this research, the subject is initially exposed to a set of patterns, usually from a single category and usually for one time only. ${ }^{2}$ Following 
this exposure, the subject is then transferred to a recognition series, which often produces false alarming to the category prototype. It seems unlikely that the degree of category abstraction afforded by a single trial (or single exposure to the patterns of a category, usually for only a few seconds per category instance) would be sufficient to promote a high degree of category learning or the integration of category instances into a stable categorical representation. Trial-by-trial performance in category abstraction research usually begins around chance, with a steady decline in errors across trials (e.g., Barresi, Robbins, \& Shain, 1975; Estes, 1986; Homa \& Cultice, 1984).

If a single trial is insufficient to produce a category representation, then false recognition must occur for reasons unrelated to category abstraction. One likely candidate is interstimulus similarity. For example, when subjects are asked to provide similarity ratings to multiple stimuli from multiple categories, even before learning has occurred, the resulting multidimensional space is structured. In fact, the category prototype is more centrally located than other instances of that category (Homa, Rhoads, \& Chambliss, 1979). It can be demonstrated that overall similarity of each pattern to the remaining patterns of a category is monotonically related to its distance to the category centroid, with near-centroid stimuli having greater similarity than far-centroid instances. Given the centrally located position of the category prototype prior to learning, false recognition of the category prototype would be expected if recognition is mediated by interitem similarity (Nosofsky, 1988), even when categories were unlearned. In effect, exemplar similarity and not an abstraction mechanism might be responsible for the false recognition of the category prototype.

The third point addresses whether the formation of a category prototype need result in its later false recognition. Although many current models of classification predict a close relationship between degree of prototype formation and likelihood of false recognition (e.g., Knapp \& Anderson, 1984; McClelland \& Rumelhart, 1986), counterexamples are easy to imagine. Looking for the perfect mate, listening to a version of your favorite symphonic selection, or tasting a favorite dish may each have in common two components: you know what the ideal is, and you are certain you have not yet experienced it. This argument assumes, of course, that the abstracted prototype is an imaginary category ideal, and that the observer treats the experienced exemplars as distortions or flaws of the ideal. Memory monitoring research (Johnson, Raye, Wang, \& Taylor, 1979; Johnson \& Suengas, 1989), which is concerned with the ability to separate imagined events from externally experienced ones, suggests that frequency estimates of an event may be partially contaminated (and elevated) by imagining the event. However, subjects in these experiments still have demonstrated a reasonable ability to distinguish between real and imagined events. In a similar vein, the category prototype may be sensed without confusing it with external reality. If this is the case, then prototype abstraction may occur without concomitant errors in recognition. This issue is addressed more fully in the General Discussion section.

Two experiments were conducted to evaluate the relationship between category classification and recognition. The major departure from previous studies was the use of a variable number of learning trials with multiple categories prior to test. In Experiment 1, subjects made both recognition and classification judgments following a variable number of learning trials ( 1 or 9 ). In Experiment 2, a wider range of learning was used $(1,6$, or 12 learning trials), and subjects made only one of these judgments. Since each category exemplar was shown once per learning trial, recognition performance could be assessed as degree of category learning was increased.

The use of multiple learning trials also permits an evaluation of a prediction by a class of exemplar-based models of classification. Nosofsky (1988) has argued that many results in classification and recognition of category patterns can be explained without recourse to explanations involving prototype abstraction. Classification of a transfer pattern is predicted to be a function of its similarity to its category members, relative to the members of alternative categories. Recognition judgments of a transfer pattern are predicted to be determined by that item's overall familiarity, measured by that item's similarity to all exemplars of all categories, summed across the relative frequency of all exemplars. According to this model, the familiarity of all transfer items, including new and unrelated foils, can only increase with increased learning trials. ${ }^{3}$ Therefore, oldness judgments for all transfer instances, including foils, might be expected to increase as well with increasing learning trials. Alternatively, familiarity but not oldness judgments may increase with increased learning trials. In this latter case, the subject may increase the threshold for oldness judgments across trials in response to the elevated familiarity for all items. These, as well as other alternative explanations, are evaluated by formal models in the General Discussion section.

\section{EXPERIMENT 1}

In Experiment 1, the subjects received either one or nine learning trials with 18 stimuli, 6 in each of three prototype categories. Half of the patterns for each category were moderate-level distortions, and half were highlevel distortions. Following learning, the subjects received a transfer test containing old, new, prototype, and foil patterns. The subjects first made a recognition judgment with a confidence rating followed by its classification. The classification judgments functioned as a control for degree of category learning: subjects receiving nine learning trials were expected to classify all transfer patterns more accurately than subjects receiving only a single trial. Given this expected outcome, the focus was upon recognition 
judgments-that is, whether oldness judgments, especially for the category prototypes, would also increase with the increased number of learning trials.

\section{Method}

Subjects. The subjects were 26 male and female students in an experimental psychology course at Arizona State University, 13 in each learning condition (one or nine learning trials). None of these students received prior instruction on categorization theory.

Materials and Apparatus. The stimuli were nine-sided forms, which have been described previously by Homa (1978). Briefly, the category prototype is defined by initially placing nine dots in a $50 \times 50$ grid and then connecting the dots in a particular order, thus forming a closed figure. Distorted exemplars of each prototype are determined by moving each of the dots of the category prototype according to a statistical decision rule and then connecting the dots with straight lines in the same order as the category prototype. Medium-level distortions have each dot move, on the average, about 2.80 Euclidean units; high-level distortions have each dot move, on the average, about 4.60 units. The patterns were drawn in black ink by a Cal-Comp plotter, photographed, and inserted in slides. A Kodak Carousel projector was used to present the stimuli on a white screen. The subjects were run in groups of 5-8 each and were seated about 10-12 ft from the screen.

Procedure. The subjects were told, in part, that the task required them to learn to classify form patterns into three groups, called A, $B$, and $C$. The procedure for each stimulus presentation was the same: a pattern was shown for $5 \mathrm{sec}$, followed by the command "record." If the subject had not yet recorded a response for that pattern (A, B, or C), he/she was instructed to do so immediately, guessing if necessary. One second following the "record" command, corrective feedback was given (e.g., "B"), followed by a $1-\mathrm{sec}$ pause, and then the carousel was advanced to the next slide. In all, each stimulus presentation took about $8 \mathrm{sec}$. The subjects in the one-trial condition received 18 different stimuli, 6 belonging to each of three prototype form classes, in a randomized order. For the subjects in the nine-trial condition, the same procedure was followed, except that the 18 stimuli were shown nine times in three different random orders. The subjects recorded their responses on prepared score sheets.
The transfer test was given immediately following learning. The subjects were told that the transfer test was similar to the learning phase in that they would again be shown patterns on the screen before them. However, there were four differences: (1) the experimenter would provide no feedback, (2) some of the patterns were new, (3) most of the patterns belonged to the same three categories as before, but some patterns were unrelated to the learning categories and could be assigned to a none category, and (4) they would first make a recognition judgment, with confidence, followed by a classification test.

A total of 54 transfer patterns was presented, each for $10 \mathrm{sec}$. Of the 54 patterns, 18 were old and 36 were new. The 18 old patterns were the original learning stimuli (6 in each category, with 3 medium-level and 3 high-level distortions). The 36 new stimuli included 18 new instances (6 per category, with 3 each at the medium- and high-level distortions), 6 prototype instances ( 2 copies of each of the 3 category prototypes), and 12 foils (patterns generated from alternative prototype categories). These patterns were shown in a single random order, with the restriction that the two copies of the same category prototype could not occur consecutively, and no more than four members of the same category could occur consecutively.

The recognition judgment required that the subject first identify the pattern as old or new, followed by a confidence judgment $(1=$ guessing, 2 = moderately certain, $3=$ certain). The classification judgment required that the subject assign the pattern either to one of the original learning groups (A, B, or C) or to a none category. The subjects recorded these double judgments (e.g., O2, C refers to a moderately certain [2] judgment of Old, with the pattern assigned to Category $\mathrm{C}$ ) on prepared score sheets.

\section{Results}

Mean percent correct classification for each type of transfer item (old, new, prototype, foil) is shown in the left panel of Figure 1 as a function of learning trials (one or nine). Generally, the effect of increased learning trials was to improve transfer performance by about $22 \%$ $\left[F(1,24)=13.34, M S_{\mathrm{e}}=27.58, p<.01\right]$. Differences in classification accuracy among the item types was sig-
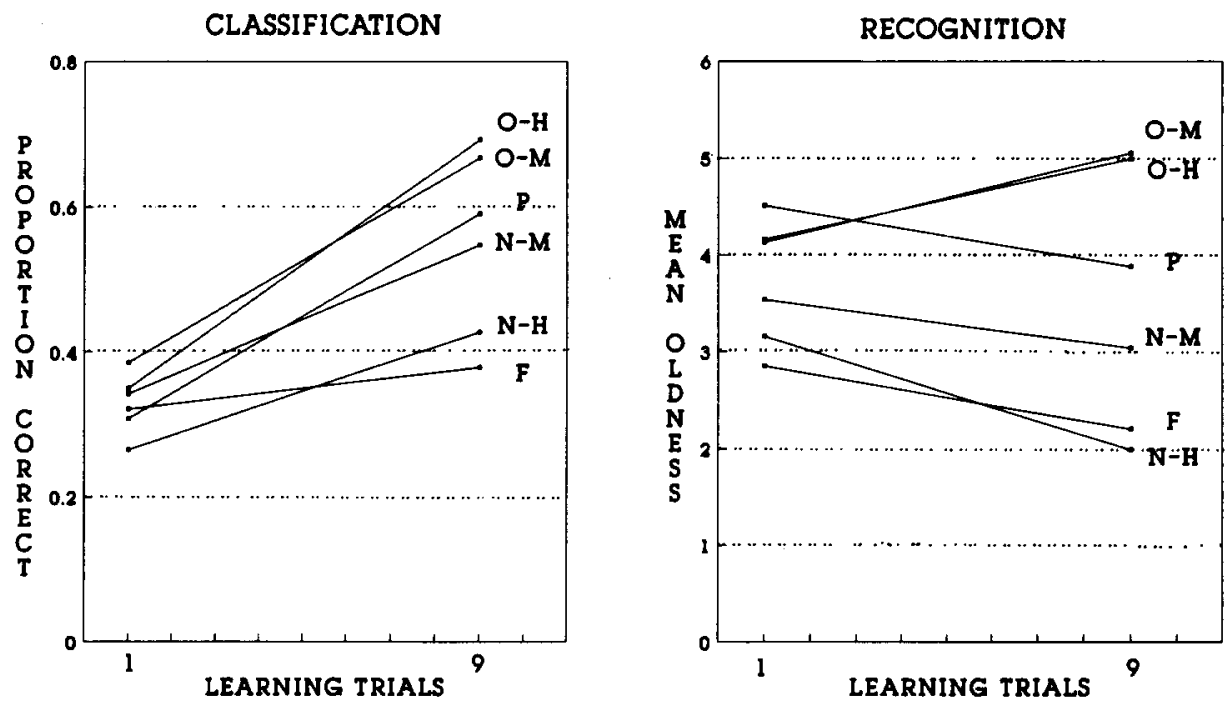

Figure 1. Transfer performance for each item type, as a function of learning trials, in Experiment 1 (O-H = old-high; O-M = old-medium; $\mathbf{N}-\mathbf{M}=$ new-medium; $\mathbf{N}-\mathbf{H}=$ new-high; $\mathbf{P}=$ prototype; $F=$ foils). 
nificant $\left[F(3,72)=3.86, M S_{\mathrm{e}}=12.21, p<.05\right]$, with improvements across trials somewhat greater for the training patterns (Old- $1=.368$; Old-9 $=.680$ ) than for the new instances $(\mathrm{New}-1=.304$; New-9 $=.487$ ) or the category prototype $(\mathrm{P}-1=.308 ; \mathrm{P}-9=.590)$. The foils were classified at a slightly improved rate after nine learning trials $(\mathrm{F}-1=.321 ; \mathrm{F}-9=0.378)$. Each of these improvements across trials was significant $(p<.05)$, except for the foils $(p>.20)$. The trials $\times$ item type interaction was marginally significant $[F(3,72)=2.27$, $\left.M S_{\mathrm{e}}=12.21, p<.10\right]$.

Separate analyses revealed that distortion level was a significant variable for new instances $[F(1,24)=5.83$, $\left.M S_{\mathrm{e}}=1.74, p<.05\right]$, but not old ones $(F<1)$. Generally, old patterns were classified equally well, regardless of distortion level (medium $=0.526$, high $=.521$ ), whereas new medium level distortions were classified more accurately $(.444)$ than high level distortions $(.346)$. The trials $\times$ distortion level was not significant $(p>$ $.20)$.

The recognition results are shown in the right panel of Figure 1. These data were converted into an oldness scale, with old $-3=6$, old $-2=5$, old $-1=4$, new $-1=3$, new $-2=2$, and new $-3=1$. The main effect of item type (old, new, prototype, foil) was significant $[F(3,72)=$ $222.59, M S_{\mathrm{e}}=1.74$ ], as was the interaction between item type and trials (one or nine) $\left[F(3,72)=10.50, M S_{\mathrm{e}}=\right.$ $1.74, p<.01]$. Overall, oldness judgments increased across trials for the training patterns $(\mathrm{O}-1=4.14 ; \mathrm{O}-9=$ 5.02) and decreased for the remaining patterns $(\mathrm{N}-1=$ $3.50, \mathrm{~N}-9=2.52 ; \mathrm{P}-1=4.51, \mathrm{P}-9=3.88 ; \mathrm{F}-1=$ $2.85 ; \mathrm{F}-9=2.20$ ), with all $p s<.05$.

A comparison of item types revealed some interesting outcomes. First, the oldness rating following one learning trial was highest for the prototype stimuli, exceeding that of new instances $[t(12)=6.87, p<.01]$ and marginally exceeding that of old instances $[t(12)=1.83, p<$ .05 , one-tailed test]. These outcomes were also reflected in the likelihood of calling these stimuli old: after one trial, old, prototype, new, and foil instances were called old on $67.5 \%, 83.3 \%, 48.7 \%$, and $34.6 \%$, respectively, of their presentations. After nine trials, oldness ratings were higher for the old instances, relative to the prototype $[t(12)=3.98, p<.01]$, with the prototype still rated higher in oldness than the new instances $[t(12)=5.95$, $p<.01]$. Again, the likelihood of calling these instances old reflected the oldness ratings, with the old, prototype, new, and foil instances called old on $85.9 \%, 61.5 \%$, $26.9 \%$, and $16.7 \%$ of the occasions. Second, distortion level was a significant variable for new instances, but not for the old patterns, on the recognition test, resulting in a significant item type $\times$ distortion level interaction $\left[F(1,24)=13.41, M S_{\mathrm{e}}=0.24, p<.01\right]$. In fact, the oldness rating for the new high-level distortions was slightly lower than the foils after nine learning trials. Third, classification accuracy after one trial was poor and near chance (overall rate $=0.325$; chance with four transfer alternatives $=0.250$ ), with performance among the stimulus types not differing from each other $(p>.20)$.

\section{Discussion}

The major results of Experiment 1 were that (1) classification performance began at near-chance levels following a single learning trial, with performance systematically improving for all transfer items (old, new, prototype, foils) with additional learning trials, (2) oldness ratings decreased with increasing learning trials for new patterns, including the category prototype, and increased for old instances, (3) oldness rating for the category prototype was highest when category learning was minimal (after one trial), and (4) although the oldness rating for the category prototype significantly decreased with learning trials, it still exceeded that for other new instances.

Before discussing the implications of these results, these findings were replicated under somewhat different conditions. First, the subjects were given either a classification or a recognition test following learning, but not both. Second, three levels of category learning $(1,6$, or 12 learning trials) were used.

\section{EXPERIMENT 2}

\section{Method}

Subjects. The subjects were 120 male and female undergraduates in an introductory psychology course at Arizona State University, 20 in each of six conditions ( 3 levels of learning $\times 2$ types of test).

Materials and Apparatus. The same type of materials (but different specific patterns) were used in Experiment 2. As before, learning and transfer was conducted in small groups (5-10). A Kodak Carousel projector was used to present stimuli.

Procedure. The general procedure was identical to that used in Experiment 1, except that the subjects received either a classification or a recognition test following 1,6 , or 12 learning trials. The composition of the learning and transfer trials mirrored that in Experiment 1; for example, the subjects classified 18 stimuli per trial in learning and received a transfer set of 54 stimuli for the recognition or classification test.

\section{Results}

Learning. Learning rates were similar for the six groups, with accuracy of classification on the initial trial ranging from 0.365 to .403 (average $=.387$, chance with three learning categories $=.333$ ), improving to $.653 \mathrm{after}$ six learning trials, and reaching 0.924 and .906 for the Classification 12 and Recognition 12 groups on the terminal learning trial.

Transfer. The left panel of Figure 2 shows the mean percent correct classification on the transfer test, as a function of number of learning trials $(1,6$, or 12$)$ and transfer item type (old, new, prototype, or unrelated). As was the case in Experiment 1, performance improved from near-chance levels after a single learning trial (.347) to moderate levels after $6(.486)$ and $12(.672)$ learning trials $\left[F(2,57)=36.05, M S_{e}=74.14, p<.01\right]$. The effect of item type was significant $[F(3,57)=20.37$, $\left.M S_{\mathrm{e}}=59.08\right]$; the item type $\times$ trial interaction was marginally significant $\left[F(6,171)=2.10, M S_{\mathrm{e}}=59.08, p<\right.$ $.06]$. In general, the effect of increased learning trials was to improve classification performance on the transfer test by about $30 \%$, being somewhat greater for the old and prototype stimuli ( $50 \%$ and $36 \%$, respectively) and some- 

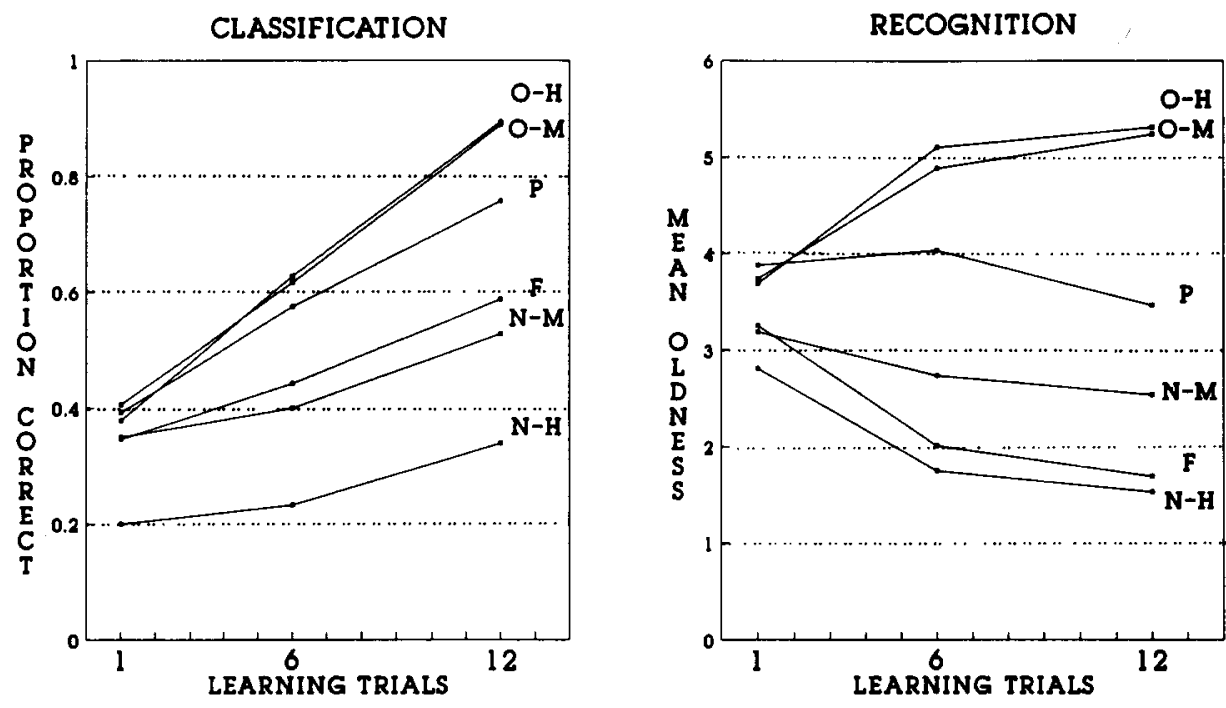

Figure 2. Transfer performance for each item type, as a function of learning trials, in Experiment 2 (O-H = old-high; $\mathbf{O}-\mathbf{M}=$ old-medium; $\mathbf{N}-\mathbf{M}=$ new-medium; $\mathbf{N}-\mathbf{H}=$ new-high; $\mathbf{P}=$ prototype; $\mathbf{F}=$ foils).

what less for new patterns and foils $(18 \%$ and $26 \%$, respectively).

The effect of distortion level was significant for new instances but not old instances, producing a significant interaction between these variables $\left[F(1,57)=21.98, M S_{\mathrm{c}}=\right.$ $1.54, p<.01]$. For old instances, the overall mean percent correct classification rate for medium- and high-level distortions was .637 and .628, respectively; for new instances, the corresponding values were .428 and .252 .

Recognition results were again converted to an oldness scale prior to analyses. The right panel of Figure 2 shows the mean oldness ratings on the recognition transfer test, as a function of learning trials and item type. An analysis of variance revealed that item type (old, new, prototype, or foil) was a significant variable $[F(3,171)=244.21$, $\left.M S_{\mathrm{e}}=0.35, p<.01\right]$, as was the item type $\times$ trial interaction $\left[F(6,171)=28.96, M S_{e}=0.35\right.$, both $\left.p s<.01\right]$. Oldness ratings for the training instances increased with increasing number of trials, whereas the remaining types generally decreased across trials. The sole exception was the category prototype, whose oldness ratings increased slightly from Trial 1 to Trial $6(p>.20)$ and then decreased from Trial 6 to Trial $12(p<.05)$. As was the case in Experiment 1, the category prototype had the highest oldness rating after a single learning trial, being significantly greater than the new instances and foils $(p<$ $.05)$. Unlike Experiment 1 , the slightly higher oldness rating for the category prototype, relative to the old instances, was not significant $(p>.10)$. A separate analysis of distortion level for old and new revealed a significant distortion level $\times$ trials interaction $(p<.01)$. This interaction took the same form as that in Experiment 1: distortion level did not affect the oldness judgments for the training stimuli $(p>.20)$, whereas the oldness judgments for new instances were significantly higher for the medium-level instances, relative to the high-level instances, especially after 1 learning trial $(p<.01)$. These data were also reflected in the likelihood of calling these instances old; for the old instances, these rates were $55.6 \%, 85.8 \%$, and $89.2 \%$, following 1,6 , and 12 learning trials, respectively. The corresponding rates were $60.0 \%, 64.2 \%$, and $51.7 \%$ for the prototype stimuli, $41.9 \%, 23.1 \%$, and $17.2 \%$ for the new instances, and $27.9 \%, 9.2 \%$, and $5.4 \%$ for the foils.

\section{Discussion}

The results of Experiment 2 replicated the major findings of Experiment 1: (1) classification accuracy began at near-chance levels following a single learning trial and systematically improved for all transfer patterns with increased learning trials; (2) oldness judgments increased across trials for the training stimuli and generally decreased across trials for all novel instances; (3) oldness judgments following a single trial were highest for the category prototype; (4) distortion level affected oldness ratings for the new patterns but not for the training instances. The only departure from the results of Experiment 1 was the slight (nonsignificant) elevation of oldness ratings for the category prototype from Trial 1 to Trial 6 . Therefore, we cannot conclude that oldness ratings for the category prototype are strictly decreasing monotonic functions of learning trials. Nonetheless, the oldness ratings following 12 learning trials were significantly lower than the ratings after 6 learning trials. Finally, the general consistency of results between Experiments 1 and 2 suggests that the double judgment required in Experiment 1 was not a significant variable.

\section{GENERAL DISCUSSION}

Previous studies (e.g., Franks \& Bransford, 1971; Metcalfe \& Fisher, 1986; Solso \& Raynis, 1979) have demonstrated that the category prototype is recognized as an old pattern, a result often interpreted as support for an ab- 
straction process. In the present experiments, the prototype was also judged as old as or older than (in terms of false-alarm rates or oldness rating) any transfer pattern, including the old instances, following a single learning trial. This outcome replicates this often-obtained finding. However, the judged familiarity of the category prototype, as well as other new instances, was reduced, not increased, by increased learning in Experiments 1 and 2 . Since classification accuracy clearly improved with additional learning trials, the question arises whether an abstraction process can explain the patterning of classification and recognition performance after one and several learning trials. We argue that the recognition performance on the category prototype after a single trial, both in the present study and in previous studies, is probably due to a simple exemplar generalization process, on the basis of similarity to the stored instances, not to a prototype abstraction process. However, interpretation of the results following multiple learning trials is less clear.

First, learning conditions likely to foster recognition of the category prototype as old need not be conducive to abstraction mechanisms. This was clearly true in both experiments, since judged oldness of the category prototype was high when degree of category learning was low. The low levels of learning after a single learning trial imply that integrative mechanisms, such as those needed for prototype abstraction, are weak or absent. ${ }^{4}$ Previous studies that have reported false recognition of the category prototype after a single learning trial failed to measure degree of learning prior to transfer. For example, Metcalfe and Fisher (1986), Neumann (1977), Solso and McCarthy (1981a, 1981b), and Solso and Raynis (1979) used a single observational trial, and no index of learning was provided. Only Metcalfe and Fisher followed learning with a transfer classification test, but the greaterthan-chance levels of classification (roughly $40 \%-70 \%$ for various transfer items) may be a misleading index of learning: $90 \%$ of the instances were minimal-level distortions, and subjects without prior exposure may reach comparable levels of classification on the transfer test. Although we cannot ascertain what level of learning occurred in these studies, we speculate that it was low.

Second, false recognition of the category prototype may be explained by mechanisms involving exemplar similarity. Because the category prototype is, by definition, likely to be centrally located among its instances, overall interstimulus similarity can be higher for the category prototype than for other instances, including the training instances. Average interstimulus similarity for any pattern then declines as degree of distortion (or distance from the centroid) increases. This inverse relationship between pattern distortion and average interstimulus similarity is highly correlated with oldness judgments in previous studies, as well as in the present one. For example, oldness judgments were highest for the category prototype, followed by new-medium and new-high distortions in all five data sets. Objective interpattern distances in the present study also reflect this continuum: the average
Euclidean distance between two medium-level distortions, between two high-level distortions, and between a mediumand a high-level distortion is $4.55,7.41$, and 5.96 units, respectively. The distance of the prototype to a mediumand high-level distortion is 2.96 and 4.83 units, respectively. The graded effect of rated oldness or familiarity with pattern distortion should obtain if abstraction does not occur, rather than the opposite.

Third, a formal exemplar-based model of classification can explain both the classification and the recognition data following a single learning trial and, to an arguable degree, the performance following multiple learning trials. The particular form of the exemplar-based model used to fit the data represents an amalgamation of previous models and is not intended as a test of any particular model; rather, the point is to demonstrate the existence of at least one model of this form that can reasonably capture the data.

\section{An Exemplar-Based Analysis of Classification and Recognition}

In most exemplar-based models (e.g., Busemeyer, Dewey, \& Medin, 1984; Medin \& Schaffer, 1978; Nosofsky, 1988), classification and recognition judgments are each mediated by interstimulus similarity. Typically, classification of a probe into a category is determined, in part, by its average within-category similarity to that category, relative to its similarity to members in the alternative categories (e.g., Busemeyer et al., 1984). Recognition typically involves the judged oldness of each instance on the basis of its similarity to the full complement of stored patterns. In Nosofsky's (1988) model, the familiarity of any item is likely to increase with increased numbers of learning trials, since instance familiarity is a multiplicative value of its similarity to other training instances and its relative frequency.

At first glance, the obtained oldness ratings, which were reduced for new instances with increased learning trials, appear to contradict the model. However, this outcome can be made consistent with model predictions by assuming that the subject sets a criterion for oldness judgments that is increased in response to the increasing familiarity produced by multiple learning trials. After a single learning trial, old, new, prototype, and foil items would have somewhat similar familiarity values, and placement of the criterion would generate moderate discrimination among these items. With increasing learning trials, the familiarity of the training patterns would increase by a disproportionate amount, relative to new patterns, resulting in enhanced discrimination between these items. This follows from the fact that familiarity is indexed by the similarity of retrieved traces and their relative frequency, and an old item will likely retrieve itself (plus related items). In contrast, a new item could retrieve other traces (but not itself). Therefore, the familiarity of new items should grow less rapidly across learning trials than should old items. It would be possible, therefore, to increase placement of the criterion with increased learning trials such 
that most of the old items would pass the criterion, but most new items would not.

The major similarity relationships were six withincategory similarities and one between-category similarity relationship: $x_{0}$, the similarity of an old instance to itself; $x_{\mathrm{mm}}$, the similarity of a medium-distortion instance to another instance of medium-level distortion; $x_{\mathrm{mh}}$, the similarity between a medium- and high-level distortion; $x_{\mathrm{hh}}$, the similarity between two high-level distortions; $x_{\mathrm{pm}}$, the similarity between the category prototype and a medium-level distortion; and $x_{\mathrm{ph}}$, the similarity between the category prototype and a high-level distortion. The similarity between an instance to a training pattern from another category is $x_{b}$.

The similarity between an item to itself was fixed at unity $\left(x_{0}=1\right)$. Once similarity values were computed to maximize classification performance, these same values were used to calculate the familiarity of the transfer items. An optimal criterion was then calculated such that familiarity values that exceeded the criterion were called old by the subject. ${ }^{5}$

For example, the classification algorithm for assigning Pattern $\mathrm{X}$ into its correct Category $\mathrm{A}$ is

$$
P(A: X \in A)=[E a /(E a+E b+E c)]\left(1-e^{-\alpha T}\right),
$$

where $E a, E b$, and $E c=$ exemplar evidence favoring Categories $\mathrm{A}, \mathrm{B}$, and $\mathrm{C}$, respectively, $T=E a+E b+E c$, and $\alpha=$ threshold for none category.

The various Eis are based on the specific similarity relationships for old, new, and prototype instances. For example, an old instance that is a medium-level distortion has an identity relationship to itself $\left(x_{0}=1\right)$, an $x_{\mathrm{mm}}$ relationship to two other old instances that are medium-level distortions, and an $x_{\mathrm{mh}}$ similarity relationship to three high-level instances. Therefore, the exemplar similarity of an old medium-level distortion to the six members in its category is

$$
E a, o m=x_{0}+2 x_{\mathrm{mm}}+3 x_{\mathrm{mh}}=1+2 x_{\mathrm{mm}}+3 x_{\mathrm{mh}} .
$$

The familiarity of each item is the sum of its similarities to exemplars in its own and other categories, indexed by its relative frequency; for example, the familiarity (FAM) of an old medium-level distortion after a single learning trial is

$$
\text { FAMom }=x_{\mathrm{o}}+2 x_{\mathrm{mm}}+3 x_{\mathrm{mh}}+12 x_{\mathrm{b}} .
$$

The $F A M$ value for each type of item (old-medium, oldhigh, etc.) is the mean value for that item, distributed with variance $k$, on a familiarity continuum, with $k$ a free parameter to be determined. Each item type for each data set is assumed to have the same variance. ${ }^{6} \mathrm{~A}$ single criterion on the familiarity continuum is then sought, such that items exceeding the criterion are called old and items having a value less than the criterion are called new.

The model was fitted to the five data sets determined by the two experiments $\times$ numbers of learning trials. Each classification data set consisted of 17 values, where each category item could be assigned to its correct category, an erroneous category, or to a none category; foils were assigned to either a none category or one of the categories. The recognition data consisted of six values, the likelihood of calling each transfer item old.

Table 1 shows the predicted and observed classification rates for the five data sets for Model 1. Overall, the obtained classification data, including erroneous classifications into alternative and none categories, are nicely captured by the model, with discrepancies between predicted and observed averaging between $2.3 \%$ to $4.5 \%$.

Table 2 shows the observed and predicted rates of calling an item old in the five data sets, on the basis of the similarity values of Model 1 , as well as the derived familiarity $(F A M)$ values for these items. Generally, these predictions are less accurate than for the classification data, with deviations between observed and predicted

Table 1

\begin{tabular}{|c|c|c|c|c|c|c|}
\hline \multirow[b]{2}{*}{ Items } & \multicolumn{2}{|c|}{$\mathrm{CC}$} & \multicolumn{2}{|c|}{ IC } & \multicolumn{2}{|c|}{$\mathrm{J}$} \\
\hline & Obs. & Pred. & Obs. & Pred. & Obs. & Pred. \\
\hline \multicolumn{7}{|c|}{ EXPERIMENT 1} \\
\hline \multicolumn{7}{|c|}{ Learning 1} \\
\hline Old-M & .385 & .389 & .504 & .456 & .111 & .155 \\
\hline Old-H & .350 & .346 & .530 & .478 & .120 & .176 \\
\hline New-M & .342 & .339 & .444 & .481 & .214 & .180 \\
\hline New-H & .265 & .266 & .487 & .517 & .248 & .217 \\
\hline Proto & .308 & .310 & .603 & .496 & .090 & .195 \\
\hline Foils & & & .679 & .779 & .321 & .221 \\
\hline \multicolumn{7}{|c|}{ Learning 9} \\
\hline Old-M & .667 & .700 & .299 & .234 & .034 & .067 \\
\hline Old-H & .692 & .664 & .231 & .251 & .077 & .085 \\
\hline New-M & .547 & .537 & .316 & .307 & .137 & .156 \\
\hline New-H & .427 & .428 & .308 & .350 & .265 & .222 \\
\hline Proto & .590 & .594 & .333 & .283 & .077 & .123 \\
\hline Foils & & & .622 & .638 & .378 & .362 \\
\hline
\end{tabular}

Observed and Predicted Classification Rates, Based on Exemplar Model of Classification, Experiments 1 and 2

\begin{tabular}{|c|c|c|c|c|c|c|}
\hline \\
\hline Old-M & .406 & .432 & .450 & .403 & .144 & .164 \\
\hline Old-H & .378 & .362 & .500 & .435 & .122 & .203 \\
\hline New-M & .350 & .340 & .478 & .445 & .172 & .215 \\
\hline New-H & .200 & .203 & .450 & .502 & .350 & .294 \\
\hline Proto & .392 & .394 & .458 & .421 & .150 & .185 \\
\hline Foils & & & .654 & .729 & .346 & .271 \\
\hline \multicolumn{7}{|c|}{ Learning 6} \\
\hline Old-M & .611 & .644 & .350 & .270 & .050 & .086 \\
\hline Old-H & .628 & .600 & .333 & .291 & .039 & .109 \\
\hline New-M & .400 & .395 & .417 & .378 & .183 & .227 \\
\hline New-H & .233 & .243 & .444 & .434 & .322 & .323 \\
\hline Proto & .575 & .579 & .367 & .301 & .058 & .120 \\
\hline Foils & & & .558 & .663 & .442 & .337 \\
\hline \multicolumn{7}{|c|}{ Learning 12} \\
\hline Old-M & .889 & .900 & .094 & .079 & .017 & .020 \\
\hline Old-H & .894 & .886 & .089 & .086 & .017 & .028 \\
\hline New-M & .528 & .534 & .200 & .180 & .272 & .286 \\
\hline New-H & .339 & .314 & .150 & .220 & .511 & .466 \\
\hline Proto & .758 & .747 & .092 & .131 & .150 & .121 \\
\hline Foils & & & .412 & .376 & .588 & .624 \\
\hline
\end{tabular}

EXPERIMENT 2

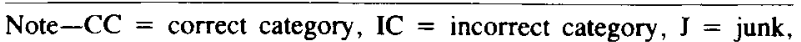
Obs. $=$ observed, Pred. $=$ predicted. 
Table 2

Observed and Predicted Recognition Rates, Based on Exemplar Model, Experiments 1 and 2

\begin{tabular}{|c|c|c|}
\hline Items & $F A M$ & $\mathrm{P}($ Old $)$ \\
\hline \multicolumn{3}{|c|}{ EXPERIMENT 1} \\
\hline \multicolumn{3}{|c|}{ Learning 1} \\
\hline $\begin{array}{l}\text { Old-M } \\
\text { Old-H } \\
\text { New-M } \\
\text { New-H } \\
\text { Proto } \\
\text { Foils }\end{array}$ & $\begin{array}{l}4.85 \\
4.51 \\
4.46 \\
3.96 \\
4.25 \\
3.92\end{array}$ & $\begin{array}{l}.684(.729) \\
.667(.643) \\
.513(.630) \\
.462(.490) \\
.833(.572) \\
.346(.479)\end{array}$ \\
\hline \multicolumn{3}{|c|}{ Learning 9} \\
\hline $\begin{array}{l}\text { Old-M } \\
\text { Old-H } \\
\text { New-M } \\
\text { New-H } \\
\text { Proto } \\
\text { Foils }\end{array}$ & $\begin{array}{r}23.24 \\
21.20 \\
15.98 \\
12.93 \\
18.04 \\
8.75\end{array}$ & $\begin{array}{l}.889(.864) \\
.829(.778) \\
.393(.467) \\
.145(.281) \\
.615(.600) \\
.167(.104)\end{array}$ \\
\hline \multicolumn{3}{|c|}{ EXPERIMENT 2} \\
\hline \multicolumn{3}{|c|}{ Learning 1} \\
\hline $\begin{array}{l}\text { Old-M } \\
\text { Old-H } \\
\text { New-M } \\
\text { New-H } \\
\text { Proto } \\
\text { Foils }\end{array}$ & $\begin{array}{l}3.68 \\
3.26 \\
3.13 \\
2.50 \\
3.44 \\
2.66\end{array}$ & $\begin{array}{l}.578(.601) \\
.533(.504) \\
.433(.474) \\
.406(.332) \\
.600(.546) \\
.279(.367)\end{array}$ \\
\hline \multicolumn{3}{|c|}{ Learning 6} \\
\hline $\begin{array}{l}\text { Old-M } \\
\text { Old-H } \\
\text { New-M } \\
\text { New-H } \\
\text { Proto } \\
\text { Foils }\end{array}$ & $\begin{array}{r}13.49 \\
12.19 \\
8.14 \\
6.19 \\
11.65 \\
5.94\end{array}$ & $\begin{array}{l}.850(.870) \\
.867(.768) \\
.317(.311) \\
.144(.140) \\
.642(.716) \\
.092(.123)\end{array}$ \\
\hline \multicolumn{3}{|c|}{ Learning 12} \\
\hline $\begin{array}{l}\text { Old-M } \\
\text { Old-H } \\
\text { New-M } \\
\text { New-H } \\
\text { Proto } \\
\text { Foils }\end{array}$ & $\begin{array}{r}16.60 \\
15.20 \\
5.29 \\
3.20 \\
8.94 \\
2.20\end{array}$ & $\begin{array}{l}.878(.916) \\
.906(.865) \\
.267(.189) \\
.078(.104) \\
.517(.449) \\
.054(.073)\end{array}$ \\
\hline
\end{tabular}

Note $-F A M=$ predicted familiarity value.

ranging from $3.9 \%$ to $10.1 \%$. Nonetheless, the model correctly predicts the diverging relationships of old and new instances across learning trials. This occurs in spite of derived familiarity values that generally increased with additional learning trials. For example, the derived familiarity of new-high items increased from 3.96 to 12.93 from Trial 1 to Trial 9 in Experiment 1, yet the likelihood of calling this item old decreased from $46 \%$ to $14 \%$. Two discrepancies may be noted. First, this model underpredicts the likelihood of calling the prototype and new instances old after 1 learning trial in Experiment 1. Second, this model must predict that old instances of lesser distortion be rated as older than those of greater distortion, regardless of number of learning trials. However, the old-medium instances were rated as less old than the old-high distortions after 6 and 12 trials in Experiment 2. Whether these deviations from prediction are specious or real is unclear. Nonetheless, the data for classification and recognition are reasonably captured by this exemplar model.

Table 3 summarizes the similarity values used to predict the classification and recognition data, as well as the estimated criterion and standard deviations for the recognition data. As indicated, the criterion is increased with additional learning trials in Experiment 1 (from 3.99 to 16.49 for 1 and 9 learning trials), whereas the criterion is increased from 1 to 6 trials in Experiment 2, and then stabilizes thereafter. The major anomaly occurs among the similarity parameters, which are not always consistent with objective interitem distance. For example, objective pattern distances would predict that derived similarity should follow the relationship: $x_{\mathrm{pm}}>x_{\mathrm{mm}}>$ $x_{\mathrm{ph}}>x_{\mathrm{mh}}>x_{\mathrm{hh}}>x_{\mathrm{b}}$. Observation of the obtained estimates indicates that this ordering was only partially followed. ${ }^{7}$

\section{Potential Influence of an Abstracted Prototype}

Each of the data sets from Experiments 1 and 2 was analyzed by a model that included a potential role of the category prototype. This analysis, similar to that reported in Busemeyer et al. (1984) and Homa et al. (1991), simply asserts that classification is based on a weighted prototype and exemplar contribution. Specifically, the classification algorithm used in Equation 1 is modified:

$P(A: X \epsilon A)=u+(1-u)[E a /(E a+E b+E c)]\left(1-e^{-\alpha T}\right)$.

When the five data sets were fitted to this model, the estimated prototype contribution to classification $(u)$ was zero for transfer performance following a single learning trial in each experiment, which provides converging support for our assertion that prototype abstraction is unlikely to occur after a single learning trial. Furthermore, the prototype contribution remained at zero following six learning trials in Experiment 2. Interestingly, the proto-

Table 3

Similarity $(x)$ and Criterion Parameters, Experiments 1 and 2

\begin{tabular}{|c|c|c|c|c|c|}
\hline \multirow[b]{2}{*}{ Item } & \multicolumn{2}{|c|}{$\begin{array}{l}\text { EXPERIMENT } 1 \\
\text { Leaming }\end{array}$} & \multicolumn{3}{|c|}{$\begin{array}{c}\text { EXPERIMENT } 2 \\
\text { Leaming }\end{array}$} \\
\hline & 1 & 9 & 1 & 6 & 12 \\
\hline$x_{0}$ & $1^{*}$ & $1 *$ & $1 *$ & $1 *$ & $1 *$ \\
\hline$x_{\mathrm{mm}}$ & .615 & .194 & .452 & .108 & .058 \\
\hline$x_{\mathrm{mh}}$ & .000 & .182 & .000 & .124 & .053 \\
\hline$x_{\text {hh }}$ & .449 & .081 & .240 & .000 & .000 \\
\hline$x_{\mathrm{pm}}$ & .406 & .137 & .397 & .279 & .800 \\
\hline$x_{\mathrm{ph}}$ & .140 & .315 & .158 & .148 & .213 \\
\hline$x_{b}$ & .218 & .054 & .148 & .055 & .009 \\
\hline$\alpha$ & .384 & 1.048 & .491 & 1.089 & 2.802 \\
\hline MAD-C & .045 & .026 & .040 & .043 & .023 \\
\hline MAD-R & .101 & .065 & .051 & .039 & .045 \\
\hline CRIT & 3.99 & 16.49 & 3.24 & 9.77 & 9.59 \\
\hline SD & 1.40 & 6.14 & 1.71 & 3.30 & 5.08 \\
\hline
\end{tabular}

Note $-x_{0}=1$ fixed parameter. MAD $=$ Mean absolute deviation between observed and predicted; $\mathbf{C}=$ classification, $\mathbf{R}=$ recognition. CRIT = criterion; $\mathrm{SD}=$ standard deviation . 
type contribution to classification was nonzero for transfer following the terminal learning trial in each experiment: In Experiments 1 and $2, u=.38$ and .33 , respectively. The possibility that a prototype contribution to classification (and recognition) emerges only following a substantial degree of learning should be viewed cautiously, since fits are somewhat overdetermined by the plethora of parameters even without the prototype parameter. Nonetheless, fits are improved by about $25 \%$ with the addition of a prototype parameter. Furthermore, the emergence of a prototype factor occurs precisely where it was found in a related experiment by Homa et al. (1991). In that experiment, instance similarity alone predicted classification after a single learning trial, with $\boldsymbol{u}=.00$. However, when the number of learning trials was increased to errorless or near-errorless criterion, the prototype contribution to classification was substantial, being somewhat greater for categories defined by more instances during learning. The potential emergence of a prototype contribution only after substantial learning in the present study, therefore, finds agreement with this outcome in a highly related study. ${ }^{8}$

\section{Summary}

Recognition of the category prototype as an old pattern, especially after a single learning trial, is probably not due to a prototype abstraction process. Neither the empirical data, which indicated minimal learning at this point, nor model analyses, which produced no evidence of a prototype influence, supported a prototype mechanism.

Although an exemplar-based model does a reasonable job predicting both recognition and classification data beyond one trial, some evidence for a prototype influence was found when multiple learning trials occurred. Here, however, the prototype was rated as less, not more, familiar. This outcome, if reliable, contradicts a number of prototype models that treat the prototype as an integration of related traces and, thus, a memorially familiar trace (e.g., Knapp \& Anderson, 1984; McClelland \& Rumelhart, 1986). For example, the distributed model of Knapp and Anderson assumes that exemplar traces, if sufficiently similar, summate to form the category prototype. However, these summed traces (the prototype representation), which would presumably foster the accurate classification of the prototype and other patterns on the transfer test, have the same reality as do memory traces of externally presented stimuli. The decreased oldness of the category prototype with increased learning trials is also inconsistent with models that deny a role of category abstraction during learning but that permit its generation and subsequent storage in response to a retrieval probe (Hintzman, 1986). The latter process would store the generated prototype as a familiar composite. We suggest, therefore, that the abstracted prototype may function as an ideal (and unfamiliar) point, at least in some circumstances, thereby having a memorial status unlike its category members. Alternatively, it may be necessary to posit a mechanism that can distinguish between internally created and exter- nally presented patterns. For example, if the subject is aware that all learning instances are high-level distortions, this knowledge could later protect the subject from identifying as familiar any subsequent test item that is minimally distorted.

An analysis of individual subject protocols suggested that subjects may differ in their categorical representations, even when multiple trials are received. We found that poor learners were more likely to rate the prototype as old. In Experiment 2, the correlation between the number of errors on the last learning trial with subsequent oldness ratings of the category prototype on the transfer test was $r(18)=+.75, p<.01 .^{9}$ This is consistent with our view that false recognition of the category prototype, rather than abstract categorical knowledge, is more likely to be found in subjects who memorize.

Finally, we have evidence (Homa \& Smith, 1992) that oldness judgments of the category prototype may increase with additional learning trials under two conditions: (1) when the training set or transfer set contain minimallevel distortions of the category prototype, and (2) when the category prototype is composed of the most frequent features that are identically contained in the learning set. Nosofsky (1991) found a high false-alarm rate after 12 trials for the category prototype that was formed from continuous features. The basis for this discrepancy with our result is unclear, but it may be due to the use of training exemplars that were highly similar to the category prototype in Nosofsky's experiment. Conceivably, a category prototype functions as an unfamiliar, ideal point when it is featurally unique and moderately dissimilar from its exemplars. We are continuing to explore these and other possibilities.

\section{REFERENCES}

Barresı, J., Robbins, D., \& Shain, K. (1975). Role of distinctive features in the abstraction of related concepts. Joumal of Experimental Psychology: Human Learning \& Memory, 1, 360-368.

Breen, T. J., Schvaneveldt, R. W. (1986). Classification of empirically derived prototypes as a function of category experience. $\mathrm{Mem}$ ory \& Cognition, 14, 313-320.

Busemeyer, J. R., Dewey, G. I. \& MEDIN, D. L. (1984). Evaluation of exemplar-based generalization and the abstraction of categorical information. Journal of Experimental Psychology: Learning, Memory, \& Cognition, 10, 638-648.

Estes, W. K. (1986). Memory storage and retrieval processes in category learning. Journal of Experimental Psychology: General, 115 , 155-174.

FraNks, J. J., \& BRANSFORD, J. D. (1971). Abstraction of visual patterns. Journal of Experimental Psychology, 90, 65-74.

HAYES-Roth, B. \& HAYES-Roth, F. (1977). Concept leaming and the recognition and classification of exemplars. Joumal of Verbal Leaming \& Verbal Behavior, 16, 321-338.

Hintzman, D. L. (1986). "Schema Abstraction" in a multiple-trace memory model. Psychological Review, 93, $411-428$.

Hома, D. (1978). Abstraction of ill-defined form. Joumal of Experimental Psychology: Human Learning \& Memory, 4, 407-416.

HoмA, D. (1984). On the nature of categories. In G. H. Bower (Ed.), The psychology of learning and motivation: Advances in research and theory (Vol. 18, pp. 49-94). New York: Academic Press.

Homa, D., Cross, J., Cornell, D., Goldman, D., \& Shwartz, S. (1973). Prototype abstraction and classification of new instances as 
a function of number of instances defining the prototype. Journal of Experimental Psychology, 101, 116-122.

HomA, D., \& Cultice, J. (1984). Role of feedback, category size, and stimulus distortion on the acquisition and utilization of ill-defined categories. Journal of Experimental Psychology: Learning, Memory, \& Cognition, 10, 83-94.

Homa, D., Dunbar, S., \& Nohre, L. (1991). Instance frequency, categorization, and the modulating effect of experience. Journal of Experimental Psychology: Learning, Memory, \& Cognition, 17, 444-458.

Homa, D., Rhoads, D., Chambliss, D. (1979). The evolution of conceprual structure. Joumal of Experimental Psychology: Human Learning \& Memory, 5, 11-23.

Hома, D., \& SMITH, C. (1992). When is the prototype recognized as old? Unpublished manuscript.

Homa, D., \& VosburGh, R. (1976). Category breadth and the abstraction of categorical information. Journal of Experimental Psychology, 2, 322-330

Johnson, M. K., RAYE, C. L., WANG, A. Y., \& TAYLoR, T. H. (1979). Fact and fantasy: The roles of accuracy and variability in confusing imaginations with perceptual experiences. Journal of Experimental Psychology: Human Learning \& Memory, 5, 229-240.

JOHNSON, M. K., \& SUENGAS, A. G. (1989). Reality monitoring judgments of other people's memories. Bulletin of the Psychonomic Sociery, 27, 107-110.

KNAPP, A., ANDERSON, I. A. (1984). Theory of categorization based on distributed memory storage. Journal of Experimental Psychology: Learning, Memory, \& Cognition, 10, 616-637.

MCClelland, J. L., \& Rumelhart, D. E. (1986). A distributed model of human learning and memory. In J. L. McClelland \& D. E. Rumelhart (Eds.), Parallel distributed processing (Vol. 2, pp. 170-215). Cambridge, MA: MIT Press.

Medin, D. L., \& SChAFFer, M. M. (1978). Context theory of classification learning. Psychological Review, 85, 207-238

Metcalfe, J., \& Fisher, R. P. (1986). The relation between recognition memory and classification learning. Memory \& Cognition, 14, 164-173.

Neisser, U. (1967). Cognitive psychology. New York: AppletonCentury-Crofts.

NeumanN, P. G. (1977). Visual prototype formation with discontinuous representation of dimensions of variability. Memory \& Cognition, 5. 187-197.

Nosofsky, R. M. (1988). Exemplar-based accounts of relations between classification, recognition, and typicality. Journal of Experimental Psychology: Learning, Memory, \& Cognition, 14, 700-708

Nosofsky, R. M. (1991). Tests of an exemplar model for relating perceptual classification and recognition memory. Joumal of Experimental Psychology: Human Perception \& Performance, 17, 3-27.

PosNer, M. I. KeELE, S. W. (1968). On the genesis of abstract ideas. Joumal of Experimental Psychology, 77, 353-363.

SHEPARD, R. N. (1987). Toward a universal law of generalization for psychological science. Science, 237, 1317-1323.

Solso, R. L. \& MCCARTHY, J. E. (1981a). Prototype formation: Central tendency model vs. attribute-frequency model. Bulletin of the Psychonomic Society, 17, 10-11.

Solso, R. L., \& MCCARThy, J. E. (1981b). Prototype formation of faces: A case of pseudo-memory. British Journal of Psychology, 72. 499-503.

Solso, R. L., \& Raynis, S. A. (1979). Prototype formation of imaged, kinesthetically and visually presented geometric forms. Journal of Experimental Psychology: Human Perception \& Performance, 5 , $701-712$

\section{NOTES}

1. Hayes-Roth and Hayes-Roth (1977) found that old instances presented 10 times in learning were generally judged older than the category prototype. However, the prototype was judged older than old instances shown once and older than any other new instances. This discrepancy with previous studies might have been fostered by an instructional set in which the rule for classification was given to half of the subjects. Unfortunately, the oldness data were not separated for this variable.

2. Metcalfe and Fisher (1986) used a single-trial observational paradigm to present three categories. However, presentation of category members was blocked, and subjects observed the instances rather than having to make categorically discriminative responses. As a consequence, degree of learning prior to transfer could not be determined.

3. An exception to this prediction can occur. If the increased number of learning trials results in dramatic decreases in similarity, then overall familiarity may decrease, rather than increase, with increased learning trials.

4. One of the reviewers questioned our calculation of chance, especially as it pertains to Trial 1 transfer performance. We would note that a random assignment of the transfer patterns into the three categories and none category results in a hit rate of .250 ; if the none category is ignored in the sorting, the hit rate improves to .333 . A bias between these extremes produces hit rates intermediate to those values above. Our only claim is that the obtained values (which ranged between .200 and .406 for individual item types in both experiments, with an overall mean of .328 in Experiment 1 and .345 in Experiment 2) are close to chance hit rates and indicative of minimal learning.

5. Parameter values were obtained by a program called STEPIT, which finds local minima of a smooth function in several parameters. Predicted recognition was determined by searching for a standard deviation and a criterion that minimized the discrepancy between obtained and predicted values.

6. Fits to the recognition data could be improved if, a priori, we knew the distributional characteristics of each item type. The assumption of equal variance for each item type is a simplifying assumption only.

7. An alternative model that assumes that similarity is exponentially related to the Euclidean distance separating the different exemplars (Nosofsky, 1988; Shepard, 1987) was also investigated, using the objective distances mentioned in the General Discussion section. Only one parameter is estimated for classification, and the resulting similarity values are forced to function lawfully with distance. Fits were fair for the classification data (mean absolute discrepancy between observed and predicted $=6.5 \%$, with a range from $4.5 \%$ to $9.0 \%$ for the five data sets) but poor for the recognition data. For Experiment 1, with 1 and 9 learning trials, the mean absolute discrepancies between observed and obtained oldness rates were $7.8 \%$ and $17.8 \%$, respectively. For Experiment 2 , with 1,6 , and 12 learning trials, the mean absolute discrepancies were $4.4 \%, 18.0 \%$, and $17.4 \%$, respectively. Generally, this version overpredicted the rate that new instances, including the category prototype, were called old, especially when more than one learning trial occurred. Interestingly, this model predicted that the category prototype should be called old at increasing likelihoods with increasing trials. Whether this model would function more successfully with psychological (e.g., multidimensionally scaled) distances (Nosofsky, 1988) is an issue currently under investigation.

8. With no additional assumptions, this model predicts recognition performance no differently from the basic exemplar model (Equation 1), in which item familiarity is determined solely by its overall summed similarity to the training instances.

9. It seems unlikely that the correlation between oldness and terminal error rate in learning for the prototype is due totally to the poorer subjects in learning simply making more errors on transfer, since this correlation was considerably lower for the old instances $(r=-.27$, $p>.05$ ). For new instances, this correlation was $r=+.60, p<.05$. We were unable to compute a comparable analysis of learning rate with oldness judgments for the category prototype for Experiment 1, because part of the learning data was inadvertently destroyed.

(Manuscript received December 11, 1991; revision accepted for publication February 3, 1993.) 